\title{
A CHARACTERIZATION OF SEMILOCAL INERTIAL COEFFICIENT RINGS
}

\author{
W. C. BROWN AND E. C. INGRAHAM
}

Abstract. A commutative ring $R$ with identity is called an inertial coefficient ring if every finitely generated $R$-algebra $A$ with $A / N$ separable over $R$ contains a separable subalgebra $S$ such that $S+N=A$, where $N$ is the Jacobson radical of $A$. Thus inertial coefficient rings are those commutative rings $R$ for which a generalization of the Wedderburn Principal Theorem holds for suitable $R$-algebras. Our purpose is to prove that a commutative ring with only finitely many maximal ideals is an inertial coefficient ring (if and) only if it is a finite direct sum of Hensel rings.

Azumaya has shown that any Hensel ring is an inertial coefficient ring [2, Theorem 33]. It follows easily [5, Proposition 3.2] that any finite direct sum of Hensel rings is an inertial coefficient ring. That an inertial coefficient ring with only finitely many maximal ideals is a direct sum of Hensel rings has been proved by Ingraham under the additional hypotheses that $R$ is noetherian and either all residue class fields of $R$ are perfect or $R$ is an integrally closed domain [5, Corollary 3.12]. Brown subsequently relaxed this final condition to $R$ being a domain whose integral closure $R^{\prime}$ in the quotient field of $R$ has the property that $R^{\prime} / \operatorname{rad}\left(R^{\prime}\right)$ is separable over $R$ [3, Theorem 3]. Our present methods simplify these earlier proofs while establishing the more general result.

All rings will contain an identity and all subrings will contain the identity of the overring. Throughout $R$ will denote a commutative semilocal ring; that is, $R$ is a commutative ring without chain conditions containing only a finite number of maximal ideals. We will call $R$ connected if it has no idempotents but 0 and 1 . Clearly every semilocal ring is a finite direct sum of connected rings. By an $R$-algebra we mean a ring along with a ring homomorphism of $R$ into the center of the ring. An $R$-algebra is called separable if it is projective as a module over its enveloping algebra [1, p. 369]. From now on, we will let $A$ denote a commutative $R$-algebra, finitely generated and faithful as an $R$-module, with Jacobson radical $N$. A separable subalgebra $S$ of

Received by the editors November 25, 1969.

AMS 1969 subject classifications. Primary 1395, 1350; Secondary 1650.

Keys words and phrases. Inertial coefficient ring, Hensel ring, inertial subalgebra. separable algebra, lifting idempotents. 
$A$ such that $S+N=A$ is called an inertial subalgebra of $A$. The basic properties of inertial subalgebras and inertial coefficient rings can be found in [5].

We begin by deriving a numerical criterion for the existence of inertial subalgebras which will be used to show that any semilocal connected inertial coefficient ring is local, that is, contains a unique maximal ideal. For any maximal ideal $\mathfrak{m}$ of $R ; A$ possesses only a finite number of maximal ideals $M$ lying over $\mathfrak{m}$, i.e. with $M \cap R=\mathfrak{m}$. For any such $M, A / M$ can be considered a field extension of $R / \mathfrak{m}$ of finite dimension $[A / M: R / \mathfrak{m}]$. Let $\mu_{\mathfrak{m}}(A)=\sum_{M}[A / M: R / \mathfrak{m}]$ where $M$ runs over those maximal ideals of $A$ contracting to the fixed maximal ideal $\mathrm{m}$ of $R$.

LEMMA 1. Let $A$ be a finitely generated, commutative, projective, faithful $R$-algebra, where $R$ is connected. If $A$ contains an inertial subalgebra $S$, then $\mu_{\mathfrak{m}}(A)=\mu_{\mathfrak{m}^{\prime}}(A)$ for any two maximal ideals $\mathfrak{m}, \mathfrak{m}^{\prime}$ in $R$.

Proof. For any maximal ideal $M$ of $A, S+M=A$ since $S+N=A$, so $S /(S \cap M) \simeq A / M$. Also distinct maximal ideals $M$ and $M^{\prime}$ of $A$ contract to distinct maximal ideals of $S$, since $S \cap M=S \cap M^{\prime}$ would give

$$
\begin{aligned}
S /(S \cap M) & =S /\left(S \cap M \cap M^{\prime}\right) \simeq\left[S+\left(M \cap M^{\prime}\right)\right] /\left(M \cap M^{\prime}\right) \\
& =A /\left(M \cap M^{\prime}\right) \simeq A / M \oplus A / M^{\prime},
\end{aligned}
$$

a contradiction. These remarks, along with the fact that every maximal ideal of $S$ is the contraction of a maximal ideal of $A$ ( $A$ is integral over $S$ ), imply that $\mu_{\mathfrak{m}}(S)=\mu_{\mathfrak{m}}(A)$. But $S / \mathrm{m} S$ is separable over the field $R / \mathrm{m}$ since $S$ is separable over $R$, implying that $S / \mathrm{m} S$ is semisimple and so is isomorphic to $\oplus \sum_{M} S /(S \cap M)$ where $M$ runs over the finite collection of maximal ideals of $A$ lying over $\mathfrak{m}$. Hence $\mu_{\mathfrak{m}}(A)$ $=\mu_{\mathfrak{m}}(S)=[S / \mathrm{m} S: R / \mathrm{m}]$. Now $S$ is a projective $R$-module [5, Proposition 2.8], so for every maximal ideal $\mathfrak{m}$ of $R, S_{\mathfrak{m}}$ has finite rank as a free $R_{\mathfrak{m}}$-module. Moreover this rank is seen to equal $[S / \mathfrak{m} S: R / \mathfrak{m}]$ $=\mu_{\mathfrak{m}}(A)$. But because $R$ is connected, the rank of $S$ localized at any maximal ideal does not depend on the choice of maximal ideal [4, Chapter 2, §4], so $\mu_{\mathfrak{m}}(A)=\mu_{\mathfrak{m}^{\prime}}(A)$, proving the lemma.

Notice that the proof of Lemma 1 holds for any ground ring $R$, regardless of how many maximal ideals it possesses.

Proposition 2. Every connected, semilocal inertial coefficient ring $R$ is local.

Proof. The proof is patterned closely after that of Theorem 3.6 of 
[5], so we omit some details. The role taken by Corollary 2.11 in that proof is here played by our Lemma 1.

Under the assumption that $R$ is connected and contains more than one maximal ideal, we construct a finitely generated, free, commutative $R$-algebra $A$ with $A / N R$-separable but such that $\mu_{\mathfrak{m}}(A) \neq \mu_{\mathfrak{m}^{\prime}}(A)$ for some maximal ideals $\mathfrak{m}, \mathfrak{m}^{\prime}$ of $R$. Thus, by Lemma $1, A$ cannot contain an inertial subalgebra, so $R$ is not an inertial coefficient ring.

We treat two cases.

Case 1. $2 \notin \operatorname{rad}(R)$. Let $m$ be a maximal ideal of $R$ with $2 \notin \mathrm{m}$. Assuming $R$ has other maximal ideals, one can choose an element $\alpha$ lying in all the maximal ideals of $R$ except m. Set $A=R[x] /\left(x^{2}-\alpha\right)$. Clearly $A$ is a finitely generated, free, commutative $R$-algebra. Furthermore, for any maximal ideal $M$ of $A$, either $A / M \simeq R /(R \cap M)$ (if $\alpha \in M$ ) or $A / M$ is an at most two-dimensional field extension of $R / \mathfrak{m}$ (if $\alpha \notin M)$. Since the characteristic of $R / \mathfrak{m}$ is not 2, we conclude that each $A / M$ and therefore $A / N$ is separable over $R$. However, one easily sees by examining $A / \mathfrak{m} A$ and $A / \mathfrak{m}^{\prime} A$ that $\mu_{\mathfrak{m}}(A)=2$ while $\mu_{\mathfrak{m}^{\prime}}(A)=1$ for any maximal ideal $\mathfrak{m}^{\prime} \neq \mathfrak{m}$. Thus $R$ is not an inertial coefficient ring by Lemma 1.

Case 2. $2 \in \operatorname{rad}(R)$. It follows from Lemma 3.10 of [5] (the assumption of noetherian therein being unnecessary) that we can assume $R$ contains a primitive cube root of 1 . Then, supposing $R$ contains more than one maximal ideal $\mathfrak{m}$, we again select $\alpha$ to be an element lying in every maximal ideal of $R$ except m. This time setting $A=R[x] /\left(x^{3}-\alpha\right)$, we see that for any maximal ideal $M$ of $A, A / M$ is a field extension of dimension one or three over $R /(R \cap M)$. (The existence of a primitive cube root of 1 is used here. See p. 88 of [5].) Since the characteristic of $R /(R \cap M)$ is two, it follows that $A / N$ is $R$-separable. One checks that $\mu_{\mathfrak{m}^{\prime}}(A)=1$ for any maximal ideal $\mathfrak{m}^{\prime} \neq \mathfrak{m}$, while $A / \mathrm{m} A$ being separable over $R / \mathrm{m}$ implies $\mu_{\mathfrak{m}}(A)=3$. Hence $R$ is not an inertial coefficient ring and the proof is complete.

Next we treat the local case. Let $R$ be a local ring with unique maximal ideal $\mathfrak{m}$. For $f \in R[x]$, denote by $\bar{f}$ the element of $(R / \mathfrak{m})[x]$ obtained from $f$ by reducing its coefficient modulo $\mathrm{m}$. $R$ is called a Hensel ring [2, p. 137] if, for every monic polynomial $f \in R[x]$ such that $\bar{f}=g_{0} h_{0}$ in $(R / \mathfrak{m})[x]$, where $g_{0}$ and $h_{0}$ are monic and relatively prime, there exist monic polynomials $g$ and $h$ in $R[x]$ with $f=g h$ and $\bar{g}=g_{0}, \bar{h}=h_{0}$. For our purposes, the important fact is that a local ring $R$ is Hensel if for every finitely generated, commutative, and faithful $R$-algebra $A$ and any ideal $I$ of $A$ with $\mathrm{m} A \subseteq I \subseteq N$, idempotents can be lifted from $A / I$ to $A$ [2, Theorem 19]. 
Proposition 3. Let $R$ be a local ring. The following statements are equivalent:

(a) $R$ is an inertial coefficient ring;

(b) Every finitely generated, commutative, faithful $R$-algebra $A$ with $A / N$ separable over $R$ is a finite direct sum of local rings;

(c) $R$ is a Hensel ring.

Proof. That (c) implies (a) is essentially the content of Theorem 33 of [2].

(a) implies (b). Let $A$ be a finitely generated, commutative, faithful $R$-algebra with $A / N$ separable. By the proof of Proposition 3.3 of [5], $A$ is itself an inertial coefficient ring since $R$ is. Now $A$ can be written as a finite direct sum of connected rings, each of which is an inertial coefficient ring [5, Corollary 3.4]. Hence each connected component of $A$ is local by Proposition 2, proving (b).

(b) implies (c). It suffices to show that for any finitely generated, commutative, faithful $R$-algebra $A$, we can lift any idempotent from $A / I$ to $A$, where $I$ is any ideal with $\mathrm{m} A \subseteq I \subseteq N$. We proceed by letting $c$ be any element of $A$ whose image $\bar{c}$ in $A / I$ is an idempotent. Consider the subalgebra $R[c]$ of $A$ generated by $c$. It is easy to verify that the natural homomorphism from $A$ onto $A / I$ when restricted to $R[c]$ gives rise to the exact sequence

$$
0 \rightarrow R[c] \cap I \rightarrow R[c] \rightarrow(R / \mathfrak{m})[\bar{c}] \rightarrow 0 .
$$

Now $(R / \mathfrak{m})[\bar{c}]$ is a homomorphic image of $(R / \mathfrak{m})[x] /\left(x^{2}-x\right)$ $\simeq(R / \mathfrak{m}) \oplus(R / \mathfrak{m})$ and so is $R$-separable. Since $(R / \mathfrak{m})[\bar{c}]$ is semisimple, $R[c] \cap I \supseteq \operatorname{rad}(R[c])$ while the opposite inclusion follows from the integrality of $A$ over $R[c]$. Therefore $R[c]$ is a finitely generated, commutative, faithful $R$-algebra such that $R[c] / \operatorname{rad}(R[c])$ is separable. Applying (b) not to $A$ but to $R[c]$, we see that $R[c]$ is a direct sum of either one or two local rings according as $R[c] / \operatorname{rad}(R[c])$ $\simeq(R / \mathfrak{m})[\bar{c}]$ is $R / \mathfrak{m}$ or $(R / \mathfrak{m}) \oplus(R / \mathfrak{m})$. In either case it is clear that there must exist an idempotent in $R[c] \subseteq A$ mapping onto $\bar{c}$. This proves Proposition 3.

As an immediate consequence of Propositions 2 and 3 we have

THEOREM. A semilocal ring (not necessarily noetherian) is an inertial coefficient ring (if and) only if it is a finite direct sum of Hensel rings.

\section{REFERENCES}

1. M. Auslander and O. Goldman, The Brauer group of a commutative ring, Trans. Amer. Math. Soc. 97 (1960), 367-409. MR 22 \#12130. 
2. G. Azumaya, On maximally central algebras, Nagoya Math. J. 2 (1951), 119-150. MR 12, 669.

3. W. C. Brown, Strong inertial coefficient rings, Michigan Math. J. 17 (1970), 73-84.

4. N. Bourbaki, Algèbre commutative. Chapitres I, II, Actualités Sci. Indust., no. 1290, Hermann, Paris, 1961. MR 36 \#146.

5. E. C. Ingraham, Inertial subalgebras of algebras over commutative rings, Trans. Amer. Math. Soc. 124 (1966), 77-93. MR 34 \#209.

Michigan State University, East Lansing, Michigan 48823 